\title{
Memory for to-be-performed tasks versus memory for performed tasks
}

\author{
JOHANNES ENGELKAMP \\ University of the Saarland, Saarbrücken, Germany
}

\begin{abstract}
Memory for subject-performed tasks-that is, for simple actions such as lifting a pen, which subjects perform overtly - is better than memory for verbal tasks - that is, when subjects only listen to the action phrases. Here I investigated whether this effect depends on actual performance or whether it also shows up when there is only an intention to perform the task. Koriat, Ben-Zur, and Nussbaum (1990) found that the intention to perform items at test enhanced free recall more than did verbal tasks. Brooks and Gardiner (1994), however, were not able to replicate this finding. In four experiments, I attempted to reconcile this discrepancy by comparing subject-performed tasks, to-beperformed tasks, and verbal tasks under different conditions. The outcome depended on whether a within-subjects design or a between-subjects design was used. In the between-subjects design, memory for subject-performed tasks was better than memory for to-be-performed tasks, and both of these led to better recall performance than did verbal tasks. In a within-subjects design, in contrast, memory for to-be-performed tasks was no different from memory for verbal tasks. These results were independent of whether the test mode was congruent or incongruent. Thus, the discrepant findings of Koriat et al. and of Brooks and Gardiner seem to be due to the design used, pointing to encoding processes as the critical variable. The present results are interpreted to show that actual performance of actions at study provides more information than does only the intention to perform actions at test.
\end{abstract}

There have been many studies in recent years on memory for subject-performed tasks (SPTs); that is, for simple actions that subjects perform overtly during the encoding phase of the experiment, such as lifting a pen, opening a book, or bending a wire. Memory for SPTs proves to be quite good. Subjects recall and recognize more action phrases when they have performed them during study than when they have only listened to them in verbal tasks (VTs; see Cohen, 1989; Engelkamp \& Zimmer, 1989, 1994, for reviews). This so-called SPT effect has essentially been attributed to the nonverbal encoding of SPTs. According to Bäckman and Nilsson (e.g., Bäckman, Nilsson, \& Chalom, 1986; Nilsson \& Bäckman, 1991), the SPT effect derives from the rich multidimensional, sensory, and motor encoding of SPTs. Engelkamp and Zimmer (1994; Zimmer \& Engelkamp, 1989b) have ascribed the SPT effect mainly to the motor encoding of SPTs because the SPT effect can also be found when the actions are performed symbolically; that is, overtly but without using real objects (see, e.g., Helstrup, 1987; Knopf, 1991). Moreover, most authors have assumed that SPTs produce a particularly discriminable memory trace that es-

This research was supported by a grant from the German Science Foundation (DFG En 124/8 and En 124/11). I am indebted to A. Kurbjuweit and $D$. Zerbst for their help in conducting the experiments and analyzing the data, and I thank R. Cohen, A. Koriat, L.-G. Nilsson, and H. Zimmer for their helpful comments on an earlier version of this article. Correspondence should be addressed to J. Engelkamp, FR 6.4 Psychologie, Universität des Saarlandes, Postfach 1150, D-66041 Saarbrücken, Germany (e-mail: engelkamp@rz.uni-sb.de). pecially facilitates recognition processes (e.g., Knopf, 1991; Nyberg, 1993; Zimmer \& Engelkamp, 1989a).

An important question regarding the SPT effect is whether it occurs only when actions are actually performed or whether it can also occur when subjects simply plan or intend to perform the action. In a study by Zimmer and Engelkamp (1984), subjects were studied in groups of 3 persons. One subject was required to assess how well actions were performed by the other 2 subjects. Both of the other subjects were always prepared to perform each action, but only 1 of them was randomly requested to perform that action. Thus, for each action phrase, 1 of the 2 subjects planned to perform the action but did not perform it, whereas the other subject planned and performed the action. The results showed that planning and enacting led to better recall than did planning only. These results support the assumption that SPTs involve an additional encoded component compared with planning. However, because no verbal task was included in Zimmer and Engelkamp, it cannot be determined whether there was any advantage for planning as such.

However, a study by Koriat et al. (1990), using a somewhat different procedure, showed that intending to perform a verbal task at test led to better memory performances than when subjects only had to recall the task verbally. In one condition, subjects learned action phrases with the intention of recalling them later verbally, whereas in another condition they learned the phrases with the intention of actually performing the actions later. Koriat et al. referred to these two conditions as memory for tobe-recalled and to-be-performed actions, respectively. 
They observed better memory when the phrases had to be performed during test than when they had to be recalled verbally. These authors concluded from the parallel positive effects of memory for to-be-performed tasks and SPTs that "the two types of tasks share the same underlying representational code... [and] perhaps the encoding of future tasks entails an internal, symbolic enactment of the tasks, which enhances memory" (p. 577). In other words, they assumed that the positive memory effect of to-be-performed actions is also due to nonverbal encoding or, more specifically, to sensorimotor or motor encoding, and that there is little difference between the intention to perform at test and actually performing actions at study. However, since they did not directly compare the instructions to perform at study with those to perform only at test, this conclusion may be premature. If Zimmer and Engelkamp's (1984) planning condition corresponds to Koriat et al.'s intention-to-perform condition, there should be an advantage of the intention to perform over a simple verbal task and a further advantage of SPTs over the intention to perform at test.

The goal of the present study was to compare the three types of tasks directly. If SPTs and to-be-performed tasks entail the same nonverbal encoding processes, they should yield comparable and better performance than do VTs. If, on the other hand, SPTs include additional processes because of the actual performance of the task during study, memory after SPTs should be better than memory after the intention to perform at test.

I know of only one study, that of Brooks and Gardiner (1994), in which SPTs, intention to perform at test, and VTs were directly compared. Although that study yielded a clear-cut advantage of SPTs over VTs and the intention to perform, it failed to find any difference between the latter two conditions. In other words, Brooks and Gardiner could not replicate the advantage of the intentionto-perform condition over verbal tasks as reported by Koriat et al. (1990). They wrote: "The failure to replicate this effect is particularly puzzling because the SPT conditions [meaning the prospective SPT conditions] in our experiments were closely modeled on the conditions used in their (third) experiment, and we deliberately chose the kind of action that Koriat et al. found gave rise to the largest effect, namely, actions involving imaginary objects" (pp. 31-32). This negative outcome of the Brooks and Gardiner study was a further reason to compare memory for SPTs, memory for intention to perform, and memory for verbal tasks.

In addition, another condition was also included in the present study, namely the combination of SPT with the intention to perform the actions again at test. In this condition, subjects perform actions at study and simultaneously form the intention to perform them again at test. This condition allows us to further test whether performing an action at study entails the encoding processes induced by the intention to perform the actions at test. If it does, combining SPTs with the intention to perform the actions at test should not lead to a better recall than SPT encoding alone.
For ease of communication, the intention to perform at test is labeled "expected motor recall," and the intention to verbally recall at test is labeled "expected verbal recall." "Test expectation" refers to the intention to verbally recall or to motorically recall (i.e., to perform the actions in recall).

The general expected outcome was that SPT/expected verbal recall should produce better memory than VT/expected motor recall, and that the latter should be better than VT/expected verbal recall. For the SPT/expected motor recall condition, the same level of performance as with SPT/expected verbal recall was expected (because encoding of SPT/expected verbal recall entails the encoding processes of SPT/expected motor recall encoding). Thus, the following pattern of results was predicted:

VT/expected verbal recall $<$ VT/expected motor recall $<$ $\mathrm{SPT} /$ expected verbal recall $=\mathrm{SPT} /$ expected motor recall.

Hence, in the following experiments, basically the two types of encoding in VTs and in SPTs were orthogonally combined with the two types of test expectation (expected verbal recall and expected motor recall).

\section{EXPERIMENT 1}

\section{Method}

Subjects. Thirty-two students of the University of the Saarland were paid for their participation.

Material. A study list of 60 action phrases was constructed. All action phrases could be easily performed when subjects were sitting at a table, such as "Comb your hair," or "Smoke a cigarette," and could be identified if performed at test. This was particularly important because the actions were performed without real objects.

For the study phase, the learning list was tape-recorded at a rate of $6 \mathrm{sec}$ per item. Half of the items were preceded by the word "enact" and half by the word "listen." Subjects were instructed to perform the actions of the phrases that followed the word "enact" and to just listen to the phrases that followed the word "listen." "Enact" and "listen" phrases occurred at random with the restriction that the same command was not repeated more than twice in succession. A second version of the study list was prepared by exchanging the commands of the items. That is, if a given item was to be enacted in Version 1, it was to be listened to in Version 2, and vice versa. Half of the subjects received Version 1 and half received Version 2. For each version, there were four different orders of items.

Design. The design involved two factors, type of encoding (VT vs. SPT) and expected testing (expected verbal recall vs. expected motor recall). The first factor was a within-subjects variable, whereas the second factor was manipulated between subjects.

Procedure. Subjects were tested individually. Before subjects were presented with the study list, they were informed that some items had to be performed (SPTs) and others only listened to (VTs), and that after the presentation of the list, a test of free recall would be administered. As to the free recall, half of the subjects were instructed that they would have to produce the phrases orally in any order; that is, to dictate them to the experimenter (verbal recall). The other half were instructed that they should learn the items in order to perform the actions in any order at test and that the experimenter would record the performed actions (motor recall).

After presentation of the study list, the subjects were requested to recall the items in a free order according to the expected test 
Table 1

\begin{tabular}{|c|c|c|c|c|}
\hline \multirow{3}{*}{$\begin{array}{c}\text { Type of } \\
\text { Encoding }\end{array}$} & \multicolumn{4}{|c|}{ Test Expectation } \\
\hline & \multicolumn{2}{|c|}{ Verbal Recall } & \multicolumn{2}{|c|}{ Motor Recall } \\
\hline & $M$ & $S D$ & $M$ & $S D$ \\
\hline \multicolumn{5}{|c|}{ Experiment 1} \\
\hline VT & .22 & .11 & .27 & .11 \\
\hline SPT & .44 & .11 & .37 & .08 \\
\hline \multicolumn{5}{|c|}{ Experiment 2} \\
\hline VT & .21 & .12 & .25 & .13 \\
\hline SPT & .43 & .12 & .38 & .13 \\
\hline
\end{tabular}

Note-VT, verbal task; SPT, subject-performed task.

mode. All subjects were requested to wait after saying or performing an item until the experimenter had recorded it and given a signal to continue. With this procedure, the temporal conditions of recall were equated between the two test modes. The experimenter asked the subjects to name the item after performing in those cases in which the experimenter could not identify the performed action. Subjects had $4 \mathrm{~min}$ available for their free recall. (In this experiment, as well as in Experiments 3 and 4, an unexpected recognition test followed after the free recall test. Because ceiling effects made the data difficult to interpret, these data are not reported.)

\section{Results}

All effects reported in this and the following experiments, if not specified otherwise, were significant at the .05 level. All post hoc pair comparisons are based on $t$ tests or on Newman-Keuls tests. Since VT/verbal recall $<\mathrm{VT} /$ motor recall $<\mathrm{SPT} /$ verbal recall $=\mathrm{SPT} /$ motor recall was the pattern of interest, these comparisons were tested post hoc whenever type of encoding and test expectation interacted significantly.

A lenient scoring criterion was adopted in order to give no advantages to the motor test mode. Only rarely did a subject who had performed the actions during test have to be asked to name an action because it was not identifiable. Table 1 shows the mean proportion of actions recalled depending on type of encoding and test expectation.

A two-way analysis of variance (ANOVA), type of encoding $\times$ type of test expectation, yielded two significant effects. SPT encoding (.41) yielded better recall than did VT encoding $\left[.24, F(1,30)=46.54, M S_{\mathrm{e}}=.0090\right]$, and the two factors interacted $\left[F(1,30)=7.21, M S_{\mathrm{e}}=\right.$ $.0090]$. Post hoc, Bonferroni corrected $t$ tests showed the following pattern:

$\mathrm{VT} /$ expected verbal recall $=\mathrm{VT} /$ expected motor recall $[t(30)=1.26]<\mathrm{SPT} /$ expected verbal recall $[t(30)=$ $4.46] \geq \mathrm{SPT} /$ expected motor recall $[t(30)=2.29]$.

Thus, the main findings were that (1) the expected motor recall advantage over verbal recall for VTs did not occur, and (2) the combination of SPT and expected verbal recall yielded better recall than did the combination of SPT and expected motor recall.

\section{EXPERIMENT 2}

\section{Method}

The design of Experiment 2 corresponded to that of Experiment 1 , except for the following modifications. Subjects learned a list of 44 phrases. These phrases were presented orally at a rate of $5 \mathrm{sec}$ per item. Before a phrase was read to the subjects, the word "enact" signaled that they should perform the action, and the word "listen" signaled that they should only listen to the phrase. Twenty-seven subjects learned the list with the intention to perform the items at test, and 27 subjects learned the same list with the intention to verbally recall the items at test. This experiment was conducted as a group experiment; therefore, free recall was administered only verbally and in a written form. Thus, subjects wrote down all the action phrases they could remember in any order they wished. Notice that for this reason, the recall mode after motor recall instructions was always incongruent; that is, subjects were instructed to expect to perform a task, but they were actually requested to recall all phrases that they could remember. However, according to the findings of Koriat et al. (1990), congruency of testing does not affect recall performance.

Fifty-four students from several psychology courses participated in this experiment.

\section{Results}

Inspection of Table 1 shows that the data for Experiment 2 are in perfect agreement with those of Experiment 1 . Again, there was an effect of type of encoding $\left[F(1,52)=64.67, M S_{\mathrm{e}}=.0124\right]$ : Free recall was better after SPTs (.41) than after VTs $(.23)$. There was also an interaction between type of encoding and test expectation that fell just short of the .05 level of significance $\left[F(1,52)=3.81, M S_{\mathrm{e}}=.0124, p<.06\right]$. In this case, post hoc Bonferroni corrected $t$ tests yielded neither a positive effect of the expectation to perform for VTs $[t(52)=$ 1.13] nor a negative effect of combining SPTs and expectation of performance $[t(52)=1.33]$. Although numerically the pattern in Experiment 2 corresponds to that of Experiment 1, the two effects were smaller than those in Experiment 1. However, recall of SPTs when verbal recall was expected was again better than recall of VTs when motor recall was expected $[t(52)=5.18]$. Thus, the following pattern of results was found in this experiment:

\section{$\mathrm{VT} /$ expected verbal $\mathrm{recall}=\mathrm{VT} / \mathrm{expected}$ motor recall $<$} $\mathrm{SPT} /$ expected verbal recall $=\mathrm{SPT} /$ expected motor recall.

\section{DISCUSSION OF EXPERIMENTS 1 AND 2}

The results of these two experiments correspond to those of Brooks and Gardiner (1994) and not to those of Koriat et al. (1990). Free recall under VT/expected verbal recall was equal to free recall under VT/expected motor recall, and free recall under SPT/expected verbal recall was better than under the two former conditions. In addition, combining SPT with the intention to perform (expected motor recall) did not enhance memory performance compared with SPT/expected verbal recall; it even interfered with it slightly.

Thus, the most important question is why Koriat et al. (1990) found a clear-cut test expectancy effect in free re- 
call, whereas Brooks and Gardiner (1994) and I did not. A closer reexamination of Koriat et al.'s study and the other experiments reveals that type of encoding was varied in the present study and in that of Brooks and Gardiner, but not in Koriat et al. In that study, only VTs were used. Moreover, in our experiments, as well as in those of Brooks and Gardiner, type of encoding was a withinsubjects variable. Brooks and Gardiner used a pure within-subjects design for the variation of VT/expected verbal recall, VT/expected motor recall, and SPT/expected verbal recall. In the present study, VT versus SPT was a within-subjects variable, and test expectation was a between-subjects variable. Thus, the variation of instructions across items, combined with the variety of different combinations of task and test expectation, might have overcharged the subjects' processing capacities.

\section{EXPERIMENT 3}

In Experiment 3, I examined whether a critical determinant of the results of the first two experiments was the simultaneous inclusion of VT and SPT as a withinsubjects variable with test expectation (expected verbal recall vs. expected motor recall) as a between-subjects variable. In Experiment 3, type of encoding and test expectation were both manipulated between subjects. Test congruency was added as a further factor. This was done for two reasons. The first reason was to approach Koriat et al.'s (1990) design as closely as possible. Koriat et al. manipulated congruency of testing mode by adding a second list in which testing was also incongruent. The second reason was to directly test whether the observed findings were only due to encoding or whether they also depended on mode of testing. Note that Koriat et al. did not find a congruency effect in their Experiment 3. Similarly, our incongruent testing of VT/expected motor recall and SPT/expected motor recall in Experiment 2 did not change the pattern of recall from that in Experiment 1, where all testing was congruent. Nevertheless, a systematic variation of test congruency is desirable, because in the present Experiment 2, testing was incongruent when there was a motor recall expectation and not when there was a verbal recall expectation.

Thus, Experiment 3 served two goals: First, it served to clarify whether the inconsistent findings of Koriat et al.
(1990) and of Brooks and Gardiner (1994) and of the present Experiments 1 and 2 were due to differences in the designs used. Second, Experiment 3 served to test whether the pattern of findings was determined by different encoding processes or whether mode of testing was also critical.

\section{Method}

Subjects. Sixty-four students of the University of the Saarland participated in this experiment. They were paid for their participation.

Material and Design. Two learning lists of 30 phrases were constructed, so that each phrase could be clearly identified if performed. Four different orders of each list were tape-recorded.

The factors in this experiment were type of encoding (VT and SPT), test expectation (expected verbal recall and expected motor recall), and congruency of testing (congruent and incongruent). The first two factors were between-subjects variables; the last was a within-subjects variable. That is, each subject learned two lists, with the first list tested under congruent conditions and the second list under incongruent conditions. The order of testing was not varied because otherwise those subjects who were first tested under incongruent conditions would have expected incongruent testing for the second list, too.

Procedure. Subjects were tested individually. Depending on the condition, the subjects were instructed to learn the phrases (1) by listening in order to verbally recall them later (VT/expected verbal recall), (2) by listening in order to perform the actions later (VT/expected motor recall), (3) by listening and then performing the actions in order to verbally recall the phrases later (SPT/expected verbal recall), or (4) by listening and performing the actions in order to perform them again later (SPT/expected motor recall). After presentation of the first list, the subjects were requested to reproduce the items in a free recall congruent with the expected test mode.

After the recall test, the subjects were told that they would be presented with a further list of new phrases, following the same instructions as those for the first list, to generate a better database. Until after list presentation, the procedure was identical to that of the first part of the experiment. Only when the recall instructions were given did the subjects hear that the recall mode was incongruent. Subjects who had expected a motor recall were requested to recall verbally, and those who had expected a verbal recall were requested to recall motorically.

\section{Results}

The relative proportions recalled correctly are summarized in Table 2. An ANOVA including the factors type of encoding, test expectation, and congruency of testing (the latter factor being a within-subjects variable) yielded

Table 2

Mean Relative Free Recall and Standard Deviations as a Function of Type of Encoding, Test Expectation (Verbal Recall, Motor Recall), and Test Congruency (Congruent, Incongruent) in Experiment 3

\begin{tabular}{|c|c|c|c|c|c|c|c|c|}
\hline \multirow{4}{*}{$\begin{array}{l}\text { Type of } \\
\text { Encoding }\end{array}$} & \multicolumn{8}{|c|}{ Test Congruency } \\
\hline & \multicolumn{4}{|c|}{ Congruent } & \multicolumn{4}{|c|}{ Incongruent } \\
\hline & \multicolumn{2}{|c|}{$\begin{array}{c}\text { Expected } \\
\text { Verbal Recall }\end{array}$} & \multicolumn{2}{|c|}{$\begin{array}{c}\text { Expected } \\
\text { Motor Recall }\end{array}$} & \multicolumn{2}{|c|}{$\begin{array}{c}\text { Expected } \\
\text { Verbal Recall }\end{array}$} & \multicolumn{2}{|c|}{$\begin{array}{c}\text { Expected } \\
\text { Motor Recall }\end{array}$} \\
\hline & $M$ & $S D$ & $\bar{M}$ & $S D$ & $M$ & $S D$ & $M$ & $S D$ \\
\hline $\mathrm{VT}$ & .34 & .10 & .43 & .07 & .33 & .09 & .40 & .11 \\
\hline SPT & .52 & .12 & .45 & .11 & .47 & .11 & 42 & .12 \\
\hline
\end{tabular}


a main effect of type of encoding $[F(1,60)=16.00$, $\left.M S_{\mathrm{e}}=.0164\right]$; recall was better after SPTs $(.47)$ than after VTs (.38). The ANOVA also yielded an interaction between type of encoding and test expectation $\left[F(1,60)=9.21, M S_{\mathrm{e}}=.0164\right]$ and a main effect of congruency of testing $\left[F(1,60)=4.7, M S_{\mathrm{e}}=.0057\right]$. Under congruent testing, subjects recalled more $(.44)$ than under incongruent testing (.41). Test congruency did not interact with the other two factors (all $F$ values $<1$ ). The most important finding was the significant interaction between type of encoding and test expectation. Subsequent Newman-Keuls tests showed that, for free recall, the ranking of performance was VT/expected verbal recall $<$ VT/expected motor recall $<$ SPT/expected verbal recall $\geq \mathrm{SPT} /$ expected motor recall, whereby the comparison of SPT/ expected verbal recall with SPT/expected motor recall just failed to attain the .05 level of significance.

Thus, the most important finding of Experiment 3 was the fact that the results replicated those of Koriat et al. (1990) in those cases in which the two designs tested the same variables. Thus, for the test expectation effect to show up in free recall, it is obviously decisive whether a withinsubjects or a between-subjects design is used for type of encoding.

\section{EXPERIMENT 4}

Before discussing the results of Experiment 3, I will briefly describe Experiment 4 . In this experiment, the positive effect of the intention to perform at test as a betweensubjects variable was replicated for the critical verbal encoding condition.

\section{Method}

Subjects. Thirty-two students of the University of the Saarland took part in this experiment. They were tested in groups of 16 subjects each.

Material and Procedure. Subjects first learned the study list of 30 action phrases. The phrases were presented orally by the experimenter at a rate of $5 \mathrm{sec}$ per phrase. Sixteen subjects were instructed to learn the phrases for verbal recall, and 16 subjects were instructed to learn them in order to perform the actions at test (i.e., for motor recall). The actual test mode for the two groups was to produce a written free recall. Thus, for the expectation of motor recall, the test was incongruent.

\section{Results}

There was a significant effect of test expectation. Free recall was better (.39) when subjects learned in order to perform than when they learned in order to verbally recall $\left[.31, F(1,30)=4.61, M S_{\mathrm{e}}=.0098\right]$. The results clearly confirm the performance-expectation effect. Also, numerically the data agree nicely with those in the corresponding conditions of Experiment 3.

\section{DISCUSSION OF EXPERIMENTS 3 AND 4}

The most important finding is that the results of Experiments 3 and 4 replicate those of Koriat et al. (1990). It is obvious that in order for the test expectation effect to show up in free recall of VTs, it is critical for type of encoding to be varied between subjects. I will reserve the discussion of this finding for the General Discussion. Here, I will discuss only the following effects, specific to Experiments 3 and 4.

1. There was a main effect of test congruency, but test congruency did not interact with the other two factors (type of encoding and test expectation).

2. In addition to the usual SPT effect, there was a clear-cut effect of the intention to perform for VTs. However, the SPT effect is clearly the stronger one. That is, SPT/expected verbal recall was not only better than VT/ expected verbal recall, but also better than VT/expected motor recall.

3. Furthermore, the same tendency was observed for an interfering effect when SPT encoding was combined with the motor recall expectation, also observed in Experiments 1 and 2. Recall performance after SPT learning decreased when this SPT learning had to be combined with the expectation to perform the actions at test again.

Because in Experiment 3 incongruent testing always followed congruent testing, some arguments will be presented showing that order of testing had no influence on the finding that congruency did not change the general pattern of recall, but that it might have influenced the level of recall under incongruent testing. Three findings support the assumption that the observed pattern of recall is indeed independent of the test mode and order of testing. First, congruency was also manipulated by Koriat et al. (1990) in their Experiment 3, and they also did not observe an interaction between test expectation and actual test mode. In contrast to our experiment, however, in their experiment, congruency was not confounded with order of testing. For each expectation group, they tested half of the suijects congruently with the expectations and half incongruently. Second, in Experiment 2 motor recall expectation was tested incongruently in a verbal recall mode, and the pattern of results of Experiment 2 was identical to that of Experiment 1, where only congruent testing was used. Third, the expectation of motor recall was also followed by a verbal test mode in Experiment 4, and the findings correspond to the congruent part of Experiment 3.

Probably only the main effect of congruency was due to the order of testing. The slightly reduced level of recall under incongruent testing conditions was possibly caused by the fact that incongruent testing followed congruent testing; that is, subjects were tested for a second learned list under incongruent testing. It is assumed that proactive inhibition built up under this condition is responsible for this effect, and not incongruent testing per se.

Thus, it can be concluded that the mode of testing is not critical for the observed pattern of recall. Furthermore, the attribution of the free recall pattern to differential encoding processes and not to differential retrieval processes is in line with the findings of two further studies in which only test mode was varied without manipulating different test expectations. The first study was by 
Saltz and Dixon (1982). In this study, subjects learned VTs and SPTs. The test was either a verbal-cued recall or a motor-cued recall with the verb of the sentence functioning as cue. Only at test did the subjects learn that they were requested either to remember the sentence belonging to the cue or to perform the action denoted by the verb before remembering the sentence. That is, the information about the mode of retrieval was given only at the time of testing. Mode of retrieval had no effect in this study. The other study was by Kormi-Nouri, Nyberg, and Nilsson (1994). They also varied type of learning (VT, SPT) and type of test (verbal, motoric). They used verbs as cues and informed the subjects about the test mode only at test. In the motor retrieval condition, subjects had to perform the action denoted by the verb before recalling the object of the phrase, much as in Saltz and Dixon. The results showed no effect of the retrieval mode. (I am ignoring a specific effect that was observed with body-related actions in Experiment 1 of Kormi-Nouri et al.)

Taken together, the null results of these studies are compatible with the assumption that the performance-atstudy effect as well as the performance-at-test effect in free recall are encoding effects, not retrieval effects.

How can the differences of encoding be explained? According to the considerations formulated in the Introduction, it is assumed that nonverbal, sensory motor encoding processes were the basis of both the positive memory effect of SPT encoding and the effect of an expected motor recall compared to a VT, for which a verbal recall was expected. Moreover, it is assumed that SPT encoding, in comparison with planning an action, such as under the expectation of motor recall, includes additional processes that come into play during the actual performance of actions during study. Again, the deviant recail patterns in Experiments 1 and 2 will be discussed in the General Discussion.

The last finding to be discussed here is the better recall of SPTs when verbal recall was expected than when motor recall was expected. This result indicates that some interference is obviously generated when the two instructions - to perform an action immediately and to plan to perform it again at test-must be followed simultaneously. This interference is ascribed to the repetition of the planning component. This argument will be elaborated upon in the General Discussion.

\section{GENERAL DISCUSSION}

It was the main goal of this study to test whether the SPT effect occurs only when actions are actually performed, or also when subjects simply intend to perform the action. Before discussing the results, I will summarize them briefly. Test congruency had no effect on the pattern of free recall performance. However, the findings depended on whether type of encoding was manipulated as a withinsubjects or a between-subjects variable. For a withinsubjects design, the pattern was VT/expected verbal recall $=\mathrm{VT} /$ expected motor recall $<\mathrm{SPT} /$ expected verbal recall $\geq$ SPT/expected motor recall. The pattern for a between-subjects design deviated only in the following aspect: VT/expected verbal recall $<\mathrm{VT} /$ expected motor recall.

First, the pattern of recall observed in the betweensubjects design will be discussed. How is it possible to explain that memory performance is best when verbal recall is expected in a subject-performed task, second best when motor recall is expected in a verbal task, and worst when verbal recall is expected in a verbal task? I assume, with Koriat et al. (1990), that the instructions to perform the actions at test instead of recalling the phrases induces nonverbal, sensorimotor encoding processes. I assume further that preparing for future actions is different from actually performing the actions at study. The intention to perform at test corresponds by and large to processes of action planning. Executing the action at study, as in SPTs, however, adds something to the planning processes. It is probably this recording of what one has done that gives the memory trace after SPT its particular discriminative power. Because the combination of SPT and expected motor recall contains a repetition of the planning process, interference is induced in this condition.

The critical question in the context of a within-subjects design is as follows: Why is recall of VTs so low when motor recall is expected? And why does this condition improve so strongly when a between-subjects design is used? I assume that two factors contribute to the low recall of VTs in a within-subjects design when motor recall is expected. First, assuming that SPTs generally lead to stronger memory traces than VTs, one could further assume that some output interference arises when a withinsubjects mixed-list design is used. The retrieval of the stronger SPT memory traces could hinder the retrieval of the weaker VT traces. A similar explanation has been advanced for the generation effect (see, e.g., Slamecka \& Katsaiti, 1987) and for the bizarreness effect of imagery (see, e.g., Einstein \& McDaniel, 1987), which also depend on type of design. Second, this interference could be enhanced when motor recall is expected. In this case, VT and SPT encoding become more similar because the planning component is involved in both tasks. As a consequence, recall of VTs is particularly low in this case.

One might argue that the simple assumption of an increasingly richer sensorimotor encoding could also explain the observed pattern of findings. Richness of encoding increases from a pure verbal task over planning to perform an action to performing an action. According to this hypothesis, interference would be due to the similarity between the involved processes. Therefore, the assumption that SPTs involve two distinct part-processes, namely planning and execution, instead of only an increasing richness of the same encoding process, seems unnecessary. I will present some evidence from other studies that show that the simple assumption of an increasing richness of an undifferentiated encoding process is not sufficient, and then concretize the assumption that planning and execution are two distinct part-processes of SPT encoding. 
If the SPT effect was based only on the amount of nonverbal sensorimotor encoding, the size of the SPT effect should increase with more sensorimotor encoding, no matter what such encoding is based on. Some findings are at variance with this hypothesis. Nyberg, Nilsson, and Bäckman (1991), for instance, observed that SPT recall without objects was better than that with objects. Moreover, the positive memory effect of actions without objects was stronger for SPTs than for VTs, and consequently the SPT effect was larger for actions without objects. This finding does not support the assumption that only the amount of sensorimotor encoding is critical. In this study, however, there was no control of action content. That is, actions without objects (e.g., clapping hands or pointing to one's nose) were different from actions with objects (e.g., flipping a coin or opening a book). Obviously, actions without objects were body related in this study. In other studies, too, body-related actions were recalled particularly well after SPTs (e.g., Cohen, Peterson, \& MantiniAtkinson, 1987; Norris \& West, 1991; Ratner \& Hill, 1991; see also Nyberg, 1993, for an elaboration of this argument). Engelkamp and Zimmer (1983) demonstrated with controlled action content that adding a real object in phrases such as "flip the coin" or "open the book" did not change the size of the SPT effect compared with a condition in which no real objects were used (the phrases were performed with imaginary objects).

Taken together, these findings suggest that the notion of sensorimotor-rich encoding is not sufficient to explain the SPT effect. Some differentiation regarding the type of sensorimotor information seems necessary. Making a distinction between planning and executing processes is one such proposal, even if it is not directly related to the role of objects in SPT encoding.

There is also physiological evidence for the assumption that different processes are involved in enactment. Without question, at a physiological level, performing an action is a complex event that involves different brain structures. The basal ganglia, for instance, seem to be responsible for perceptual-motor integration (Manetto \& Lidsky, 1989). Conceivably, they might allow for the gating of perceptual inputs during motor activity. The motor cortex itself is considered to be a trigger center rather than a planning center for movement because activity in this brain center reliably increases about $50 \mathrm{msec}$ before the first sign of electrical activity in the muscles (Deecke, Scheid, \& Kornhuber, 1969). The supplementary motor cortex, which is located just anterior to the motor cortex, is assumed to be involved in the planning and production of movements (Wiesendanger, 1987). In contrast to the motor cortex, electrical activity in this part of the brain can be detected as long as $1 \mathrm{sec}$ before movement begins (Deecke et al., 1969).

Evidence for the planning role of this brain center also comes from studies in which blood flow in the brain is monitored through the use of positron emission tomography (PET) scans. PET scans show that the supplemen- tary motor cortex as well as the motor cortex itself are highly active when subjects carry out sequences of finger movements. However, when the finger movements are only imagined, blood flow to the supplementary motor cortex remains high, but blood flow to the motor cortex returns to normal levels (Roland, Larsen, Lassen, \& Skinhoj, 1980).

These and other physiological findings (see Jeannerod, 1988; Rosenbaum, 1991, for reviews) speak in favor of the assumption that processes underlying the planning of actions are qualitatively different from those involved in the execution of actions, and - at least as far as the underlying brain structures are concerned-cannot be exchanged for each other. It seems therefore at least very plausible to claim that the encoding processes involved in planning to perform actions are different from those involved in the actual performance of actions.

\section{REFERENCES}

Bäckman, L., Nilsson, L.-G., \& Chalom, D. (1986). New evidence on the nature of the encoding of action events. Memory \& Cognition, 14, 339-346.

Brooks, B. M., \& Gardiner, J. M. (1994). Age differences in memory for prospective compared with retrospective subject-performed tasks. Memory \& Cognition, 22, 27-33.

Cohen, R. L. (1989). Memory for action events: The power of enactment. Educational Psychology Review, 1, 57-80.

Cohen, R. L., Peterson, M., \& Mantini-Atkinson, T. (1987). Interevent differences in event memory: Why are some events more recallable than others? Memory \& Cognition, 15, 109-118.

Deecke, L., Scheid, P., \& Kornhuber, H. H. (1969). Distribution of readiness potential, premotion positivity, and motor potential of the human cerebral cortex preceding voluntary finger movements. $E x$ perimental Brain Research, 7, 158-168.

Einstein, G. O., \& MCDAniel, M. A. (1987). Distinctiveness and the mnemonic benefits of bizarre imagery. In M. A. McDaniel \& M. Pressley (Eds.), Imagery and related mnemonic processes (pp. 78-102). New York: Springer-Verlag.

ENGELKAMP, J., \& ZimmeR, H. D. (1983). Zum Einfluß von Wahrnehmen und Tun auf das Behalten von Verb-Objekt-Phrasen. Sprache \& Kognition, 2, 117-127.

Engel Kamp, J., \& Zimmer, H. D. (1989). Memory for action events: A new field of research. Psychological Research, 51, 153-157.

Engelkamp, J., \& Zimmer, H. D. (1994). The human memory. Seattle: Hogrefe \& Huber.

Helstrup, T. (1987). One, two, or three memories? A problemsolving approach to memory for performed acts. Acta Psychologica, 66, 37-68.

JEANNEROD, M. (1988). The neural and behavioral organization of goal-directed movements. Oxford: Oxford University Press.

KNOPF, M. (1991). Having shaved a kiwi fruit: Memory of unfamiliar subject-performed actions. Psychological Research, 53, 203-211.

Koriat, A., Ben-Zur, H., \& Nussbaum, A. (1990). Encoding information for future action: Memory for to-be-performed versus memory for to-be-recalled tasks. Memory \& Cognition, 18, 568-578.

Kormi-Nouri, R., NyberG, L., \& Nilsson, L.-G. (1994). The effect of retrieval enactment on recall of subject-performed tasks and verbal tasks. Memory \& Cognition, 22, 723-728.

ManetTo, C., \& Lidsky, T. I. (1989). The effect of movements on caudate sensory responses. Neuroscience Letters, 96, 295-299.

NiLSSON, L.-G., \& BäCKMAN, L. (1991). Encoding dimensions of subject-performed tasks. Psychological Research, 58, 212-218.

NORRIS, M. P., \& WEST, P. L. (1991). Age differences in the recall of actions and cognitive activities: The effects of presentation rate and object cues. Psychological Research, 53, 188-194. 
NYBERG, L. (1993). The enactment effect: Sudies of a memory phenomenon. Unpublished doctoral dissertation, University of Umeå. Sweden.

Nyberg, L., Nilsson, L.-G., \& Bäckman, L. (1991). A component analysis of action events. Psychological Research, 53, 219-225.

Ratner, H. H.. \& Hill, L. (1991). The development of children's action memory. When do actions speak louder than words? Psychological Research, 53, 195-202.

Roland, P. E., Larsen, R., Lassen, N. A., \& Skinhoj, F. (1980). Supplementary motor area and other cortical areas in organization of voluntary movements in man. Journal of Neurophysiology, 43, 118-136.

Rosenbaum, D. A. (1991). Human motor control. San Diego: Academic Press.

SALTZ, E., \& DiXon, D. (1982). Let's pretend: The role of motoric imagery in memory for sentences and words. Journal of Experimental Child Psychology, 34, 77-92.

Slamecka, N. J., \& Katsaiti, L. T. (1987). The generation effect as an artifact of selective displaced rehearsal. Journal of Memory \& Language, 26, 589-607.
WIESENDANGER, M. (1987). Initiation of voluntary movements and the supplementary motor area. In H. Heuer \& C. Fromm (Eds.), Generation and modulation of action patterns (pp. 3-13). Berlin: Springer-Verlag.

Zimmer, H. D., \& Engelkamp, J. (1984). Planungs- und Ausführungsanteile motorischer Gedächtniskomponenten und ihre Wirkung auf das Behalten ihrer verbalen Bezeichnungen. Zeitschrift für Psychologie, 192, 379-402.

Zimmer, H. D., \& ENGElKamp, J. (1989a). Does motor encoding enhance relational information? Psychological Research, 51, 158167.

Zimmer, H. D., \& Engelkamp, J. (1989b). One, two or three memories: Some comments and new findings. Acta Psychologica, 70, 293-304.

(Manuscript received July 18, 1995; revision accepted for publication November 6,1995 .) 